\title{
A Quantitative Approach to Drug Dosing in Chronic Kidney Disease
}

\author{
Ali J. Olyaei Jessica L. Steffl \\ Division of Nephrology and Hypertension, Oregon State University and Oregon Health and Science University, \\ Portland, Oreg., USA
}

\section{Key Words}

Chronic kidney disease $\cdot$ Drug pharmacokinetics $\cdot$ Dialysis • Drug dosing

\begin{abstract}
Chronic kidney disease (CKD) is increasing at an alarming rate. Medication prescribing in this growing population is especially difficult. Many pharmacological agents or their metabolites are eliminated unchanged through the kidney. Drug dosing in CKD is challenging as most patients have a number of comorbid conditions. Patients with CKD take pharmacological agents with potential for drug interactions. Most patients also have alterations to the normal functioning of a number of different organs or systems (e.g. heart, liver, gastrointestinal system) which affect pharmacokinetics of commonly used drugs in CKD. Pharmacokinetic behaviors of most drugs are highly variable in patients with CKD. In addition, pharmacological management of patients with CKD is imprecise and requires estimating renal function, applying clinical judgment and, if available, therapeutic drug monitoring to provide adequate pharmacotherapeutic concentrations to optimize pharmacodynamic response while minimizing toxicities. For drugs that are removed through the renal system unchanged, a dosing modification should be considered according to patient- and drug-specific factors. Renal replacement therapy and dialysis remove phar-
\end{abstract}

macologic agents extensively, and thus a replacement dose is needed to avoid therapeutic failure. By applying a quantitative approach, health care providers can improve pharmacotherapeutic outcomes while reducing adverse drug reactions.

Copyright ๑ 2011 S. Karger AG, Basel

\section{Introduction}

Pharmacokinetic and pharmacodynamic properties of many medications are greatly affected by kidney disease. Whether acute or chronic, kidney disease and resulting uremia have a global effect on the body. Therefore, when prescribing medications in patients with kidney disease, multiple factors must be taken into account: drug absorption, distribution, metabolism, and excretion of active or toxic metabolites may all be affected by kidney disease and uremia. The complexity of drug prescribing in this patient population increases as kidney disease worsens and particularly in renal replacement therapy (hemodialysis or peritoneal dialysis, PD) [1-3].

Chronic kidney disease (CKD) strongly influences drug excretion. Many medications require dosage adjustments in kidney disease in order to ensure efficacy and prevent toxicity. Pharmacologic agents may have active metabolites that are renally excreted in addition to un-

\section{KARGER}

Fax +4161306 1234

E-Mail karger@karger.ch

www.karger.com
(C) 2011 S. Karger AG, Basel

0253-5068/11/0313-0138\$38.00/0

Accessible online at:

www.karger.com/bpu
Prof. Ali J. Olyaei

Division of Nephrology and Hypertension, Oregon State University and

Oregon Health and Science University, 3181 SW Sam Jackson Park Road

Mail Code CR9-4, Portland, OR 97201 (USA)

Tel. +1 503494 8132, E-Mail olyaeia@ ohsu.edu 
changed drug. In kidney disease, the metabolism and elimination of these agents is reduced. Accumulation of active drug or metabolite may lead to complications, particularly in medications with a narrow therapeutic index [4]. Therapeutic drug monitoring may be necessary to ensure adequate drug levels while assessing for accumulation and potential toxicity. However, drug levels are not always available, and therefore assessment of pharmacokinetic and pharmacodynamic characteristics of the medication will assist in preventing adverse events. Considering all characteristics of a medication is essential to maintain a safe balance in this patient population [5].

Intracellular and extracellular fluid balance is maintained primarily by the kidneys; therefore, changes in the fluid balance of the body can affect the pharmacokinetics of drugs by affecting the volume distributions. The volume of distribution (Vd) of the pharmacologic agents in the body changes as the fluid balance of the body changes. In renal impairment and particularly in patients requiring renal replacement therapy, large shifts in fluid distribution can occur compared to patients with normal renal function [6]. The changes in Vd combined with potential for drug clearance in dialysis can increase difficulty of medication prescribing in this patient population. Due to the pathophysiologic changes in CKD and the presence of comorbid conditions, patients require an average of eleven medications [7]. Studies have shown an increase in mortality in patients with CKD as the number of medications prescribed increase [8]. Thus, careful medication management in this patient population is essential.

This paper will discuss the use of medications in patients with CKD and how to optimize pharmacotherapy in patients on dialysis while preventing the potential risk for toxicity.

\section{Initial Patient Assessment}

A thorough patient assessment is essential to reduce the potential for complications in the treatment of patients with CKD. The past medical history should include current medications, drug allergies and any previous adverse drug events. Due to the incidence of comorbid conditions in patients with CKD, such as cardiovascular disease or metabolic disorders, patients take a large number of medications for disease management. The number of medications coupled with the pharmacokinetic and pharmacodynamic changes in these patients creates a threefold higher incidence of adverse drug events com- pared to patients with normal renal function. Individualization of pharmacotherapy to each patient's unique characteristics can reduce the potential for adverse effects. In addition, minimizing the number of medications required may provide additional risk reductions. Medications may be chosen in order to concurrently treat two conditions, for example using beta-antagonists for hypertension and tachycardia. Therefore, a thorough review of the patient's medication list combined with their problem list may allow for optimization of drug therapy.

\section{Principles of Altered Pharmacokinetics in Renal Failure}

As previously discussed, pharmacokinetic parameters are altered in CKD. Bioavailability, Vd, protein binding, and biotransformation are all affected by impaired renal function.

\section{Bioavailability}

The bioavailability of a medication is the fraction of the total dose of a drug of that reaches systemic circulation. The major variables contributing to bioavailability are the route of drug delivery and the rate of administration. Intravenous medication administration places the agent directly into systemic circulation, and because the entire dose is present in the vasculature, it is $100 \%$ bioavailable. Other routes of administration are susceptible to multiple factors contributing to potential decreased absorption. Oral, intramuscular and subcutaneous injections must pass an additional compartment or multiple compartments before reaching systemic circulation and therefore have decreased bioavailability. Agents may not be completely absorbed or have the potential to be eliminated in the liver by the first pass effect. Dosage adjustments may be necessary when converting a drug from its intravenous to oral form.

Renal disease can significantly affect drug adsorption, decreasing the bioavailability of oral medications. Multiple factors may contribute to the decreased absorption. Kidney disease and commonly associated co-morbid conditions have a combined effect on various aspects of absorption [9, 10]. For example, altered gastrointestinal function such as gastroparesis, as a side effect of diabetes mellitus and/or aging, decreases absorption. Additionally, uremia-induced vomiting may prevent drug from remaining long enough in the gastrointestinal tract to be absorbed. Another example is patients with congestive heart failure or cirrhosis combined with renal failure 
have edematous gastrointestinal tract due to fluid overload causing decreased oral bioavailability. Typically, furosemide given intravenously has $100 \%$ bioavailability, and orally the average bioavailability is approximately $50 \%$. However, when gut edema is present, the bioavailability can decrease to as low as $10 \%$, requiring drastic dosage adjustments to gain similar diuretic effects [11]. Finally, concomitant administration of medications in renal failure can alter the absorption in several ways. Phosphate binders and histamine 2-receptor antagonists, often prescribed in renal failure, can change gastric $\mathrm{pH}$, altering medication absorption [12]. Insoluble complexes may form when certain medications (e.g. aluminum or calcium-containing phosphate binders) are given along with antibiotics or other iron-containing supplements. These complexes can decrease absorption and slow gut motility [13].

\section{Volume of Distribution}

The Vd is a proportion of the volume of pharmacological agent in the body compared to the amount of drug in the serum. It is a hypothetical volume that does not represent a specific compartment in the body but rather a potential distribution throughout the body. Vd is represented by the following equation:

$$
\mathrm{Vd}=\frac{\mathrm{A}_{\mathrm{B}}}{\mathrm{C}}
$$

$\mathrm{Vd}$ is equal to the amount of drug in the body $\left(\mathrm{A}_{\mathrm{B}}\right)$ divided by the concentration in the serum $(C)$. There is an inverse relationship between serum concentrations and the Vd. For example, medications with a small Vd will maintain higher serum concentrations and vice versa. Therefore, determining the dose necessary to reach a specific systemic drug level often requires knowledge of the $\mathrm{Vd}$. This information can be useful when targeting a specific area in the body in which an agent would need to reach a certain concentration.

The kidney plays a major role in affecting the $\mathrm{Vd}$ of a pharmacologic agent. Drug distribution and protein binding are both affected by kidney disease. Once a drug reaches systemic circulation, it is initially distributed to high blood flow organs then later to those with lower blood flow such as fat, bone and skin. Each agent has a unique profile for the rate and extent of distribution throughout the body. These characteristics determine the amount of pharmacologic action each medication will exert. Fluid balance also greatly affects the Vd of medications. As the extracellular fluid increases, as in patients with edema or ascites, the Vd increases. The increase in distribution will therefore decrease the serum concentra- tion. In instances of volume depletion, the Vd will decrease, thus increasing the serum concentration. Changes in extracellular fluid volume have the greatest effect on hydrophilic compounds or those with low Vd $(<0.7 \mathrm{ml} /$ $\mathrm{kg})$. Consideration of fluid status is important when dosing this category of medications [14].

\section{Protein Binding}

Many pharmacologic agents are bound, to varying extents, to proteins in the plasma. The unbound portion of drug molecules has the ability to exert the pharmacological effects, whereas bound molecules are inactive. Kidney disease and uremia can affect the ability of plasma proteins to bind with medications. Uremic molecules and other organic wastes have the ability to bind to plasma proteins and displace the agent. The rise in unbound drug molecule, or free fraction of drug, increases the potential pharmacologic action of the medication. For example, phenytoin, a highly protein-bound (98\%) drug, requires dosage adjustments based on the extent of available protein binding and due to its narrow therapeutic index, dosage adjustments may be required and are guided by drug assays. However, most drug assays give a total concentration rather than differentiating between free and bound drug levels. In patients with kidney disease, it is essential to specifically monitor the free fraction of drug rather than the total concentration [15].

\section{Biotransformation}

Biotransformation refers to the process of chemical change to a pharmacologically active agent from a parent compound to a drug metabolite. Most drugs metabolize to a significantly more water soluble agent with less lipophilic properties. A number of drugs metabolize to a pharmacologically active agent with a pharmacotherapeutic profile similar to parent compounds (morphine, glyburide). These active metabolites might be excreted actively through urine. Although the parent drugs do not accumulate in CKD, the active metabolites might accumulate in these patients and metabolite-related adverse drug reactions should be considered when these agents are used. It is well known that CKD affects how the human body metabolizes and eliminates drugs [16]. Patients with kidney disease have slower nonrenal drug clearance and a prolonged half-life compared with patients with normal renal function. Recently, Zhang et al. [17] reported a longer half-life was observed in patients with kidney disease for number of new approved drugs that undergo nonrenal elimination. These observations had been reported for many commonly used drugs in CKD patients. 
Table 1. Common inhibitors of the P450 CYP3A4 and CYP2C9 enzyme systems

\begin{tabular}{llll}
\hline CYP3A4 inhibitors & & & CYP2C9 inhibitors \\
\hline Amiodarone & Fluvoxamine & Nelfinavir & Amiodarone \\
Cannabinoids & Grapefruit juice & Norfloxacin & Azole antifungals \\
Clarithromycin & Indinavir & Propoxyphene & Chloramphenicol \\
Cyclosporine & Itraconazole & Quinine & Cimetidine \\
Danazol & Ketoconazole & Ritonavir & Fluoxetine \\
Delavirdine & Omeprazole & Saquinavir & Fluvastatin \\
Diltiazem & Metronidazole & Sertraline & Fluvoxamine \\
Erythromycin & Mibefradil & Troleandomycin & Metronidazole \\
Fluconazole & Miconazole & Verapamil & Omeprazole \\
Fluoxetine & Nefazodone & Zafirlukast & Ritonavir \\
& & & Sulfaphenazole \\
& & & TMP/SMX \\
& & & Zafirlukast
\end{tabular}

For example, patients with kidney disease are at greater risk of morphine intoxication. Morphine is metabolized to morphine-6-glucuronide and morphine-3-glucuronide [18]. In CKD patients, plasma concentrations of these agents are higher than patients with normal renal function. Midazolam is another example, as critical care patients with CKD are at greater risk of prolonged sedation from midazolam. Although midazolam is metabolized through the liver, $\alpha$-hydroxymidazolam, an active metabolite, has been shown to accumulate in CKD [19].

Some drugs need to be converted to an active form to have any pharmacologic effect. For example most angiotensin converting enzyme inhibitor agents are prodrugs and need to be activated before they exert any pharmacological effects. Although most drug metabolism occurs in the liver, other organs such as the kidneys, small intestine and lungs may contribute to drug metabolism. Phase I and II reactions are the most important chemical reactions seen in drug biotransformation [20,21]. The phase I drug metabolism involves oxidation, reduction, and hydrolysis. During oxidation reactions, oxygen is inserted into a drug molecule. The most clinically significant oxidation enzymes include cytochrome P450. Phase II reactions, called synthetic or conjugation reactions, involve the attachment of another chemical group to the drug, resulting in a chemical with greater water solubility and renal elimination. Most drugs undergo both reactions during their biotransformation to produce a metabolite that will be easily excreted in the urine. Renal disease and accumulation of uremic toxins downregulate expression of many cytochrome P450 enzyme activities and increase the risk of drug toxicity. The process leading to this phe- nomenon is poorly understood, but most likely related to gene and protein expression [16]. Many drugs may inhibit or induce the cytochrome P450 system and be a source of significant drug interaction. These drug interactions may result in either a reduced pharmacodynamic effect or increased risk of adverse drug reactions [22]. It is important to recognize the pharmacokinetic behavior of these agents and know which drugs act as cytochrome P450 enzyme substrates, inducers, or inhibitors so as to prevent clinically significant interactions from occurring. Avoiding coadministration, anticipating potential toxicities and/or adjusting pharmacotheraputic agent can provide optimal pharmacodynamic response with minimal toxicities (table 1) [23].

Finally, a variety of other factors may influence drug metabolism: polymorphism, age, liver disease, smoking, alcohol and diet have been shown to alter the expression of the cytochrome P450 system [24].

\section{Elimination}

The glomerular filtration rate (GFR) is closely correlated with pathology and functional ability of the kidney. Acute or chronic kidney injury, loss of nephrons and reduced renal function correspond with a decrease in glomerular function and tubular secretions. For drug dosing in patients with CKD, GFR is useful in determining renal function and dosage adjustments. There are number of methods available to estimate GFR or overall health of the kidney. The most common practical method to estimate GFR is estimating creatinine clearance (Clcr). Cre- 
atinine is an endogenous molecule that is produced at a constant rate. Various factors can interfere with creatinine production and clearance: a low-protein diet, liver disease, decreased muscle mass and some drugs may interfere with creatinine reading and falsely estimate renal function. Drugs such as glucocorticoids and androgens increase the creatinine production and/or urea production. Cimetidine and trimethoprim inhibit renal tubular creatinine secretion and can also cause falsely increased serum creatinine $(\mathrm{Scr})$. Nonsteroidal anti-inflammatory agents may impair the kidney's ability to excrete free water.

Despite their limitations, Scr and estimated Clcr remain the most practical approach to estimate GFR. Measuring 24-hour Clcr is not practical or convenient to patients and subject to error during the 24-hour collection. Radiocontrast isothalamate or isotope infusions are not ideal methods, as both methods are more invasive, expensive and carry an increased risk of adverse drug reactions [25]. The Cockcroft and Gault equation is widely used to estimate the Clcr [26]. The Cockcroft and Gault formula includes the variables of age (years), ideal body weight (IBW; $\mathrm{kg})$, and stable Scr $(\mathrm{mg} / \mathrm{dl})$ and calculates the $\mathrm{Scr}(\mathrm{ml} / \mathrm{min})$ :

$$
{ }^{*} \text { Clcr }=\frac{(140-\text { age }) \times \text { IBW }}{72 \times \text { Scr }}
$$

In women, the result should be multiplied by 0.85 to correct for smaller muscle mass than men and, therefore, less creatinine produced. For patients with acute kidney injury and low urine outputs, Clcr should be assumed to be $<10 \mathrm{ml} / \mathrm{min}$. More importantly, Cockcroft-Gault should not be used in this setting. The Cockcroft-Gault equation tends to overestimate the GFR. It was developed in 1965 and shows a lack of patient weight in the laboratory report for renal function; therefore, the Modification of Diet in Renal Disease (MDRD), a new formula to estimate renal function, has been advocated for assessment of renal function. The MDRD equation, or specifically eGFR (MDRD4), estimates GFR rather than Clcr and, as such, may provide a more accurate estimate of renal function. The major advantage of the MDRD equation is that it is standardized to a body surface area (BSA) of $1.73 \mathrm{~m}^{2}$. The MDRD GFR uses the Scr in mg/dl [27]. The MDRD Study prediction equation can be expressed as follows:

$$
\text { GFR } \left.=186.3 \cdot \mathrm{Scr}^{-1.154} \cdot \mathrm{Age}^{-0.203} 1.212 \text { [black }\right] \cdot 0.742 \text { [female] }
$$

The MDRD equation can be used for individual drug dose adjustment when the BSA is considered (GFR = MDRD $\times$ BSA $/ 1.73 \mathrm{~m}^{2}$ ) [28].
GFR $=175.6 \cdot \mathrm{Scr}^{-1.154} \cdot \mathrm{Age}^{-0.203} 1.212$ [black $] \cdot 0.742$ [female $]$

While the primary MDRD equation was developed from patients with GFR $<60 \mathrm{ml} / \mathrm{min}$, the new MDRD/ CKD-EPI equation has now been modified to include patients with different stages of CKD [28].

$$
\begin{aligned}
& G F R=141 \cdot \min (\mathrm{Scr} / \kappa, 1)^{\alpha} \cdot \max (\mathrm{Scr} / \kappa, 1)^{-1.209} . \\
& 0.993^{\text {Age }} \cdot 1.159[\text { black }] \cdot 1.018[\text { female }]
\end{aligned}
$$

Cystatin C is an endogenous biomarker of GFR which is produced by all nucleated cells and filtered freely without any tubular secretion [29]. Cystatin C production is constant and independent of age, gender, race, weight or muscle mass. However, inflammatory conditions and hyperthyroidism may interfere with cystatin C production. The GFR can be estimated from serum cystatin C according to this equation [30]:

$$
\text { GFR Cystatin } C=\frac{100}{C y s t C(m g / l)}-14
$$

Although recently MDRD has been advocated for estimating Clcr and drug dosing in CKD [31], this method has not been validated or tested for pharmacologic agent dosage adjustment in CKD [32]. Finally, the MDRD equation has been reported to be less accurate in populations with mild kidney disease or obese patients [29].

\section{Loading Dose Determination}

In patients with normal renal function, steady state drug concentrations are achieved after approximately 5 half-lives. A loading dose often is given to achieve therapeutic plasma concentration rapidly for a drug with a long half-life. In patients with CKD, steady-state plasma or desired plasma concentration is delayed if a loading dose is not given. Regardless of renal function, patients with CKD should receive a similar loading dose as patients with normal renal function in order to achieve a rapid therapeutic dose. The following formula can be used to calculate the loading dose with $\mathrm{Vd}$ in $1 / \mathrm{kg}$, ideal body weight in $\mathrm{kg}$, Cp is the desired plasma concentration in $\mathrm{mg} / \mathrm{l}$ :

$$
\mathrm{LD}=\mathrm{Vd} \times[\mathrm{Cp}]
$$

For drugs with highly lipophilic properties, the actual body weight should replace ideal body weight. 
Table 2. Therapeutic drug monitoring in CKD

\begin{tabular}{|c|c|c|c|}
\hline Drug name & When to draw sample & Therapeutic range & How often to draw levels \\
\hline $\begin{array}{l}\text { Aminoglycosides (conventional } \\
\text { dosing) gentamicin, tobramycin, } \\
\text { amikacin }\end{array}$ & $\begin{array}{l}\text { Trough: immediately prior to dose } \\
\text { Peak: } 30 \text { min after a } 30 \text { - to } 45 \text {-min } \\
\text { infusion }\end{array}$ & $\begin{array}{l}\text { Gentamicin and tobramycin: } \\
\text { Trough: } 0.5-2 \mathrm{mg} / \mathrm{l} \\
\text { Peak: } 5-8 \mathrm{mg} / \mathrm{l} \\
\text { Amikacin: } \\
\text { Peak: } 20-30 \mathrm{mg} / \mathrm{l} \\
\text { Trough: }<10 \mathrm{mg} / \mathrm{l}\end{array}$ & $\begin{array}{l}\text { Check peak and trough with } 3 \text { rd dose } \\
\text { For therapy }<72 \mathrm{~h} \text {, levels not necessary } \\
\text { Repeat drug levels weekly or if renal } \\
\text { function changes }\end{array}$ \\
\hline $\begin{array}{l}\text { Aminoglycosides ( } 24 \text {-hour dosing) } \\
\text { gentamicin, tobramycin, amikacin }\end{array}$ & Obtain random drug level $12 \mathrm{~h}$ after dose & $0.5-3 \mathrm{mg} / \mathrm{l}$ & $\begin{array}{l}\text { After initial dose } \\
\text { Repeat drug level in } 1 \text { week or if renal } \\
\text { function changes }\end{array}$ \\
\hline Carbamazepine & Trough: immediately prior to dosing & $4-12 \mu \mathrm{g} / \mathrm{ml}$ & $\begin{array}{l}\text { Check 2-4 days after first dose or change } \\
\text { in dose }\end{array}$ \\
\hline Cyclosporine & Trough: immediately prior to dosing & $150-400 \mathrm{ng} / \mathrm{ml}$ & Daily for first week, then weekly \\
\hline Digoxin & $12 \mathrm{~h}$ after maintenance dose & $0.8-2.0 \mathrm{ng} / \mathrm{ml}$ & $\begin{array}{l}\text { 5-7 days after first dose for patients } \\
\text { with normal renal and hepatic function; } \\
15-20 \text { days in anephric patients }\end{array}$ \\
\hline Enoxaprin & $4 \mathrm{~h}$ after $2 \mathrm{nd}$ or $3 \mathrm{rd}$ dose & $0.7-1.1$ units $/ \mathrm{ml}$ & Weekly and as needed \\
\hline Lidocaine & $8 \mathrm{~h}$ after i.v. infusion started or changed & $1-5 \mu \mathrm{g} / \mathrm{ml}$ & As needed \\
\hline Lithium & $\begin{array}{l}\text { Trough: before a.m. dose at least } 12 \mathrm{~h} \\
\text { since last dose }\end{array}$ & $\begin{array}{l}\text { Acute: } 0.8-1.2 \mathrm{mM} \\
\text { Chronic: } 0.6-0.8 \mathrm{mM}\end{array}$ & As needed \\
\hline Phenobarbital & Trough: immediately prior to dosing & $15-40 \mu \mathrm{g} / \mathrm{ml}$ & $\begin{array}{l}\text { Check } 2 \text { weeks after first dose or change in } \\
\text { dose } \\
\text { Follow-up level in } 1-2 \text { months }\end{array}$ \\
\hline $\begin{array}{l}\text { Phenytoin } \\
\text { Free phenytoin }\end{array}$ & Trough: immediately prior to dosing & $\begin{array}{l}10-20 \mu \mathrm{g} / \mathrm{ml} \\
1-2 \mu \mathrm{g} / \mathrm{ml}\end{array}$ & $\begin{array}{l}\text { 5-7 days after first dose or after change in } \\
\text { dose }\end{array}$ \\
\hline $\begin{array}{l}\text { Procainamide } \\
\text { NAPA (n-acetyl procainamide), } \\
\text { a procainamide metabolite }\end{array}$ & $\begin{array}{l}\text { Trough: immediately prior to next dose } \\
\text { or } 12-18 \mathrm{~h} \text { after starting or changing an } \\
\text { infusion } \\
\text { Draw with procainamide sample }\end{array}$ & $\begin{array}{l}4-10 \mu \mathrm{g} / \mathrm{ml} \\
\text { Trough: } 4 \mu \mathrm{g} / \mathrm{ml} \\
\text { Peak: } 8 \mu \mathrm{g} / \mathrm{ml} \\
10-30 \mu \mathrm{g} / \mathrm{ml}\end{array}$ & As needed \\
\hline Sirolimus & Trough: immediately prior to next dose & $10-20 \mathrm{ng} / \mathrm{dl}$ & Weekly for first month, then as needed \\
\hline Tacrolimus & Trough: immediately prior to next dose & $5-10 \mathrm{ng} / \mathrm{ml}$ & Daily for first week, then weekly \\
\hline $\begin{array}{l}\text { Valproic acid } \\
\text { (divalproex sodium) }\end{array}$ & Trough: immediately prior to next dose & $40-100 \mu \mathrm{g} / \mathrm{ml}$ & $\begin{array}{l}\text { Check } 2-4 \text { days after first dose or change } \\
\text { in dose }\end{array}$ \\
\hline Vancomycin & $\begin{array}{l}\text { Trough: immediately prior to dose } \\
\text { Peak: } 60 \text { min after a } 60 \text { min infusion }\end{array}$ & $\begin{array}{l}\text { Trough: } 10-20 \mathrm{mg} / \mathrm{l} \\
\text { Peak: } 25-40 \mathrm{mg} / \mathrm{l}\end{array}$ & $\begin{array}{l}\text { With 3rd dose (when initially starting } \\
\text { therapy, or after each dosage adjustment) } \\
\text { For therapy }<72 \mathrm{~h} \text {, levels not necessary } \\
\text { Repeat drug levels if renal function changes }\end{array}$ \\
\hline
\end{tabular}

\section{Maintenance Dose Determination}

For drugs that are mainly eliminated unchanged through urine, the maintenance dosage should be adjusted in two ways: prolong the dosing interval or dosage reduction.

Dosage interval extension allows for adequate peak concentrations but may risk subtherapeutic trough levels. Dosing reduction may provide for more constant drug levels but increases the risk of toxicity from higher plasma trough concentrations.

A Quantitative Approach to Drug Dosing in CKD

\section{Therapeutic Drug Monitoring}

For medication with a narrow therapeutic index, therapeutic drug monitoring can be beneficial despite dosage adjustments according estimated renal function. Whenever possible after changes in renal function or addition of another agent with potential nephrotoxicity, therapeutic drug concentration should be repeated. Therapeutic drug monitoring is valuable, it is expensive and not available for most drugs. Therapeutic drug monitoring in addition to clinical pharmacotherapeutic response can help 
health care providers to minimize toxicities while improving efficacy. It is important to remember that there is an overlap between toxicity, therapeutic plasma concentration and therapeutic failure. Toxicity and adverse drug reaction may occur despite appropriate plasma drug concentration. For example, despite proper plasma concentration levels, concomitant administration of vancomycin and an aminoglycoside can increase the risk for nephrotoxicity of both agents [33]. Examples of pharmacologic agents that can be monitored in CKD are shown in table 2.

\section{Drug Removal by Dialysis}

For drugs that are removed through the kidney unchanged, a dosage adjustment is required during acute or CKD. However, some of these agents also could be removed during the dialysis. Hemodynamically stable patients require intermittent hemodialysis (IHD) or PD. However, critically ill patients with unstable hemodynamics may require continuous renal replacement therapy (CRRT) [34]. Currently, the most frequently used modality for CRRT is continuous venovenous hemodialfiltration. Compared to dialysis, which uses mostly diffusion and hemofiltration which uses primarily high convective rate, continuous venovenous hemodialfiltration uses both diffusion and convection. Patients on renal replacement therapy are at a risk of both underdosing and toxicities. IHD requires a high blood flow rate $(200-450 \mathrm{ml} /$ $\mathrm{min}$ ) and dialysate flow (400-600 $\mathrm{ml} / \mathrm{min})$ [35]. In IHD, drug removal by diffusion is based on concentration gradients and drug removal more closely related to the blood and dialysate flow rates. This rapid solute movement through high-flux membranes encourages drug removal and necessitates a dose replacement flowing IHD. During IHD, the majority of drugs are removed primarily by the process of diffusion across the dialysis membrane. Most drugs with molecular weight (MW) $<500 \mathrm{Da}$ can be removed from the plasma to the dialysate. Numbers of high-flux dialyzers have been developed to improve solute removal during IHD. These dialyzers have larger pores, and pharmacologic agents with a higher MW weight can easily pass through these membranes. For example, vancomycin with an MW of approximately 1,400 $\mathrm{Da}$ can be removed significantly during dialysis with newer membranes. Today, most CRRT use high-flux membranes in patients at high risk of underdosing and therapeutic failure.
In general, drugs with large volumes of distribution are less likely to be removed by dialysis compared to drugs with a small Vd. Even when a drug is removed primarily unchanged through kidney, if the $\mathrm{Vd}$ is large enough, only small amounts of the drugs are removed during dialysis. Drugs with a large Vd are dispersed into adipose tissue and have extensive tissue binding. Drugs with a $\mathrm{Vd}<0.7 \mathrm{ml} / \mathrm{kg}$ are removed more extensively than drugs with a $\mathrm{Vd}>0.7 \mathrm{ml} / \mathrm{kg}$. Vd is less of an issue in CRRTx.

Another important factor that may affect drug removal during dialysis is protein binding as only free and unbound drugs can be removed during dialysis. Drugs with a high percentage of protein binding have a low dialyzability, while drugs with low protein binding may cross dialysis membranes much easier. Protein binding of more than $80 \%$ provides a substantial barrier to drug removal by convection or diffusion. In PD, the peritoneal membrane is used as the dialyzing membrane, which is much less effective compared to hemodialysis at removing pharmacologic agents. In PD, drug removal occurs as the result of diffusion and concentration gradients. However, in PD during acute peritonitis a significant amount of drug binds to proteins and can be removed in peritoneal effluents [36-38].

\section{Conclusion}

CKD can affect nearly every organ or organ system in the body. Patients with CKD require a careful and thorough evaluation when medications are being prescribed. Assessing the pharmacokinetic and pharmacodynamic properties of medications, along with specific patient characteristics, can help to ensure optimal patient outcomes while lowering the risk for toxicity.

References paired renal function. Clin Pharmacol Ther 2009;86:483-489.

2 Aymanns C, Keller F, Maus S, Hartmann B, Czock D: Review on pharmacokinetics and pharmacodynamics and the aging kidney. Clin J Am Soc Nephrol 2010;5:314-327.

-3 Churchwell MD, Mueller BA: Drug dosing during continuous renal replacement therapy. Semin Dial 2009;22:185-188.

4 Vilay AM, Churchwell MD, Mueller BA: Clinical review: drug metabolism and nonrenal clearance in acute kidney injury. Crit Care 2008;12:235. 
5 Olyaei AJ, Bennett WM: Drug dosing in the elderly patients with chronic kidney disease. Clin Geriatr Med 2009;25:459-527.

-6 Macedo E, Bouchard J, Soroko SH, Chertow GM, Himmelfarb J, Ikizler TA, et al: Fluid accumulation, recognition and staging of acute kidney injury in critically-ill patients. Crit Care 2010;14:R82.

$\checkmark 7$ Manley HJ, McClaran ML, Overbay DK, Wright MA, Reid GM, Bender WL, et al: Factors associated with medication-related problems in ambulatory hemodialysis patients. Am J Kidney Dis 2003;41:386-393.

-8 Manley HJ, Cannella CA, Bailie GR, St Peter WL: Medication-related problems in ambulatory hemodialysis patients: a pooled analysis. Am J Kidney Dis 2005;46:669-680.

9 Verbeeck RK, Musuamba FT: Pharmacokinetics and dosage adjustment in patients with renal dysfunction. Eur J Clin Pharmacol 2009; 65:757-773.

10 Verbeeck RK: Pharmacokinetics and dosage adjustment in patients with hepatic dysfunction. Eur J Clin Pharmacol 2008;64:11471161.

-11 Bellomo R, Prowle JR, Echeverri JE: Diuretic therapy in fluid-overloaded and heart failure patients. Contrib Nephrol 2010;164:153-163.

-12 Dong K, Quan DJ: Appropriately assessing renal function for drug dosing. Nephrol Nurs J 2010;37:304-308.

13 Rifkin DE, Winkelmayer WC: Medication issues in older individuals with CKD. Adv Chronic Kidney Dis 2010;17:320-328.

- 14 Hassan Y, Al Ramahi R, Abd AN, Ghazali R: Drug use and dosing in chronic kidney disease. Ann Acad Med Singapore 2009;38: 1095-1103.

15 Lacerda G, Krummel T, Sabourdy C, Ryvlin P, Hirsch E: Optimizing therapy of seizures in patients with renal or hepatic dysfunction. Neurology 2006;67(suppl 4):S28-S33.

-16 Nolin TD: Altered nonrenal drug clearance in ESRD. Curr Opin Nephrol Hypertens 2008; 17:555-559.
17 Zhang Y, Zhang L, Abraham S, Apparaju S, Wu TC, Strong JM, et al: Assessment of the impact of renal impairment on systemic exposure of new molecular entities: evaluation of recent new drug applications. Clin Pharmacol Ther 2009;85:305-311.

18 Davison SN, Mayo PR: Pain management in chronic kidney disease: the pharmacokinetics and pharmacodynamics of hydromorphone and hydromorphone-3-glucuronide in hemodialysis patients. J Opioid Manag 2008;4:335-344.

19 Spina SP, Ensom MH: Clinical pharmacokinetic monitoring of midazolam in critically ill patients. Pharmacotherapy 2007;27:389398.

20 Guengerich FP, Rendic S: Update information on drug metabolism systems - 2009 part I. Curr Drug Metab 2010;11:1-3.

-21 Rendic S, Guengerich FP: Update information on drug metabolism systems - 2009 part II: summary of information on the effects of diseases and environmental factors on human cytochrome P450 (CYP) enzymes and transporters. Curr Drug Metab 2010;11: 4-84.

22 Izzo AA, Ernst E: Interactions between herbal medicines and prescribed drugs: an updated systematic review. Drugs 2009;69:17771798.

23 Boobis A, Watelet JB, Whomsley R, Benedetti MS, Demoly P, Tipton K: Drug interactions. Drug Metab Rev 2009;41:486-527.

24 Wang J: CYP3A polymorphisms and immunosuppressive drugs in solid-organ transplantation. Expert Rev Mol Diagn 2009;9: 383-390.

25 Narva AS: Assessment of kidney function for drug dosing. Clin Chem 2009;55:1609-1611.

26 Cockcroft DW, Gault MH: Prediction of creatinine clearance from serum creatinine. Nephron 1976;16:31-41.

$\checkmark 27$ Levey AS, Atkins R, Coresh J, Cohen EP, Collins AJ, Eckardt KU, et al: Chronic kidney disease as a global public health problem: approaches and initiatives - a position statement from Kidney Disease Improving Global Outcomes. Kidney Int 2007;72:247-259.

28 Stevens LA, Levey AS: Use of the MDRD study equation to estimate kidney function for drug dosing. Clin Pharmacol Ther 2009; $86: 465-467$
9 Spruill WJ, Wade WE, Cobb HH III: Continuing the use of the Cockcroft-Gault equation for drug dosing in patients with impaired renal function. Clin Pharmacol Ther 2009;86:468-470.

30 Tidman M, Sjostrom P, Jones I: A Comparison of GFR estimating formulae based upon s-cystatin $\mathrm{C}$ and s-creatinine and a combination of the two. Nephrol Dial Transplant 2008;23:154-160.

31 Levey AS, Stevens LA, Schmid CH, Zhang YL, Castro AF III, Feldman HI, et al: A new equation to estimate glomerular filtration rate. Ann Intern Med 2009;150:604-612.

>32 Dowling TC, Matzke GR, Murphy JE, Burckart GJ: Evaluation of renal drug dosing: prescribing information and clinical pharmacist approaches. Pharmacotherapy 2010;30: 776-786.

33 Goetz MB, Sayers J: Nephrotoxicity of vancomycin and aminoglycoside therapy separately and in combination. J Antimicrob Chemother 1993;32:325-334.

34 Schetz M: Drug dosing in continuous renal replacement therapy: general rules. Curr Opin Crit Care 2007;13:645-651.

35 Choi G, Gomersall CD, Tian Q, Joynt GM, Freebairn R, Lipman J: Principles of antibacterial dosing in continuous renal replacement therapy. Crit Care Med 2009;37:22682282

36 Bourquin V, Ponte B, Saudan P, Martin PY: Drugs dosing in intensive care unit during continuous renal replacement therapy (in French). Nephrol Ther 2009;5:533-541.

37 Gilmartin C: Pharmacist's role in managing anemia in patients with chronic kidney disease: potential clinical and economic benefits. Am J Health Syst Pharm 2007;64(suppl 8):S15-S22.

>38 Decker BS, Mueller BA, Sowinski KM: Drug dosing considerations in alternative hemodialysis. Adv Chronic Kidney Dis 2007; 14:e17-e26. 